\title{
BMJ Individual empowerment in overweight Open and obese patients: a study protocol
}

\author{
Pierluigi Struzzo, ${ }^{1}$ Raffaella Fumato, ${ }^{2}$ Silvia Tillati, ${ }^{3}$ Anita Cacitti, ${ }^{2}$ Fabrizio Gangi, ${ }^{1}$ \\ Alessia Stefani, ${ }^{1}$ Alessia Torcutti, ${ }^{1}$ Lucia Crapesi, ${ }^{1}$ Gianni Tubaro, ${ }^{1}$ \\ Matteo Balestrieri ${ }^{4}$
}

To cite: Struzzo P, Fumato $\mathrm{R}$, Tillati S, et al. Individual empowerment in overweight and obese patients: a study protocol. BMJ Open 2013;3: e002669. doi:10.1136/ bmjopen-2013-002669

- Prepublication history for this paper are available online. To view these files please visit the journal online (http://dx.doi.org/10.1136/ bmjopen-2013-002669).

Received 31 January 2013 Revised 20 April 2013 Accepted 22 April 2013

This final article is available for use under the terms of the Creative Commons Attribution Non-Commercial 2.0 Licence; see http://bmjopen.bmj.com

\footnotetext{
${ }^{1}$ Region Friuli Venezia Giuia, Regional Centre for the Training in Primary care, Monfalcone, Italy ${ }^{2}$ Department of Mental Health, Local Health Unit 'Medio Friuli', Udine, Italy ${ }^{3}$ Department of Economic and Statistical Sciences, Università degli Studi di Udine, Udine, Italy ${ }^{4}$ Department of Psychiatry, Teaching Hospital of Udine and DISM, University of Udine, Udine, Italy
}

Correspondence to Dr Pierluigi Struzzo; pierluigi. struzzo@uniud.it

\section{ABSTRACT}

Introduction: Obesity is a growing health problem in Europe and it causes many diseases. Many weightreducing methods are reported in medical literature, but none of them proved to be effective in maintaining the results achieved over time. Self-empowerment can be an important innovative method, but an effectiveness study is necessary. In order to standardise the procedures for a randomised controlled study, a pilot study will be run to observe, measure and evaluate the effects of a period of selfempowerment group treatment on overweight/obese patients.

Methods: and analysis Non-controlled, experimental, pilot study. A selected group of patients with body mass index $>25$, with no severe psychiatric disorders, with no aesthetic or therapeutic motivation will be included in the study. A set of quantitative and qualitative measures will be utilised to evaluate the effects of a self-empowerment course in a 12 month time. Group therapy and medical examinations will also complete this observational phase. At the end of this pilot study, a set of appropriate measures and procedures to determine the effectiveness of individual empowerment will be identified and agreed among the different professional figures. Results will be recorded and analysed to start a randomised controlled trial to evaluate the effectiveness of the proposed methodology.

Ethics and dissemination: This protocol was approved by the local Ethics Committee of Udine in March 2012. The findings of the trial will be disseminated through peer-reviewed journals, national and international conference presentations and public events involving the local administrations of the towns where the trial participants are resident.

Trial Registration: http://www.clinicalstrials.gov identifier NCT01644708.

\section{BACKGROUND}

According to WHO Europe, overweight and obesity are the growing health problems in Europe (in 2011, 52\% of the adult population is overweight, of which $17 \%$ is obese). ${ }^{12}$ In Italy, in 2011, the per cent of Italian obese was $10 \%$, with an increase, in respect to

\section{ARTICLE SUMMARY}

\section{Article focus}

- Our hypothesis is that self-empowerment helps people in improving the quality of life both in terms of physical and psychical well-being and in terms of weight loss, maintaining the results achieved over time.

Key messages

- The determinants of overeating should be considered.

- Self-empowerment should be considered in future weight-reducing programmes.

Strengths and limitations of this study

- It proposes the assessment of a new methodology that involves and motivates patients.

- Much of the success depends on the single expert taking care of the groups.

- There is the need to standardise the coping measures.

2001, of $1.6 \%{ }^{3}$; on including the overweight Italians, the per cent rises to $40.2 \%{ }^{4}$

The proportion of obesity varies with age: the Cardiovascular Epidemiological Observatory recorded that in Italy, between 1998 and 2002, within the general population (35-74 years), $17 \%$ of the men and $21 \%$ of the women were obese, while $50 \%$ of the men and $34 \%$ of the women were overweight; in the senior population (6574 years), $20 \%$ of the men and $32 \%$ of the women were obese, while $50 \%$ of the men and $40 \%$ of the women were overweight. ${ }^{5}$

In particular, $30 \%$ of the women in menopause were obese and $39 \%$ were overweight. ${ }^{5}$

Obesity causes many diseases-cardiovascular, type 2 diabetes, obstructive sleep apnoea, obesity hypoventilation syndrome, nonalcoholic steatosis, hepatitis, hypertension, dyslipidaemia, gastro-oesophageal reflux disease, asthma, venous stasis, severe urinary incontinence, disabling arthropathy, depression or a serious deterioration in quality of life. ${ }^{6}$ 
Apart from genetic and endocrinological causes, obesity can be related to an inability to change health behaviours because of a low control over stress factors in the patient's life. ${ }^{7}$

With regard to therapy, Epicentro states that "the treatment consists of a reduction of the body weight, under close medical supervision, often at specialised centres and in the maintenance of a weight appropriate for one's height thanks to proper nutrition." 8

Diet, which is often studied in specific cases, should be supported by a suitable physical activity and possibly by behavioural therapy. According to the US guidelines, ${ }^{9}$ in more complex cases, pharmacological therapies can be used, based on dexphenfluramine, sibutramine or phentermine/phenfluramine, for periods ranging from 6 months to 1 year. ${ }^{10}$ Otherwise, surgery is the only recommended treatment in extreme cases for those patients suffering from acute obesity (BMI $>35$ ) and other associated pathologies, that is, in patients having a high mortality risk, or who do not respond to other treatments.

\section{METHODS/DESIGN}

\section{Theoretical background}

Many methods are reported in medical literature, but none of them proved to be effective in maintaining the results achieved over time. ${ }^{11}$

Motivational change remains a fundamental step towards the maintenance of a new lifestyle. Strengthening personal capabilities and self-esteem seem to be key strategies in motivating persons for change and facilitating their coping capacity. ${ }^{12}$

Our institution, through an non-governmental organisation (NGO) called 'Diamo Peso al Benessere' (Let's give weight to wellbeing), has recently introduced a new methodology based on self-empowerment of overweight/obese patients. Major stress is put on increasing self-esteem and coping capacities. Group discussion of individual psycho-socio-economical situation is conducted by an empowerment expert. Emphasis is given to the maximisation of personal resources and future planning capacity.

In a 11 years' time (2001-2011), more than 594 patients, allocated into 45 groups, according to the annual therapeutic approach promoted by the NGO 'Diamo Peso al Benessere', were reported to have lost and maintained a considerable amount of weight (average BMI from 35.43 to 32.17 , with an overall 9.2\% reduction, $8.97 \mathrm{~kg}$ each) (tables 1 and 2). A 5-year follow-up on 149 patients showed the maintenance and an increased reduction of the weight (table 3 ).

Those results could lead to a real clinical change and have a possible high impact on people's health; however, since the scientific methodology was not duly recorded and standardised, the planning of an effectiveness study was decided on the next 80 included patients.

The aim of this pilot study is, then, to observe, measure and evaluate the clinical effects, over a period of 1 year, of a self-empowerment group treatment on overweight/obese patients.

\section{Conceptual background}

The concept of empowerment is defined in a sufficiently broad way by Rappaport. ${ }^{13}$ Empowerment is described as a process focused on action, through which persons, organisations and communities acquire mastery over their own lives. ${ }^{14} 15$

Self-empowerment is a social process recognising, promoting and strengthening the patient's ability to satisfy his own needs, involving a mobilisation of the resources needed in order to feel in control of his own life. ${ }^{16} 17$ This personal power grows with self-understanding and the change must take place on the individual level. Furthermore, self-empowerment depends not only on the need to develop a sense of choice and control but also on the need to (i) feel secure and connected and (ii) develop a sense of meaning and coherence. ${ }^{18} 19$

In the context of our mental health institution, we utilise the concept of self-empowerment in which the person's power is considered essential for the

Table 1 Mean BMI at baseline and after 1 year of self-empowerment group treatment (data for all patients and according to BMI category at baseline)

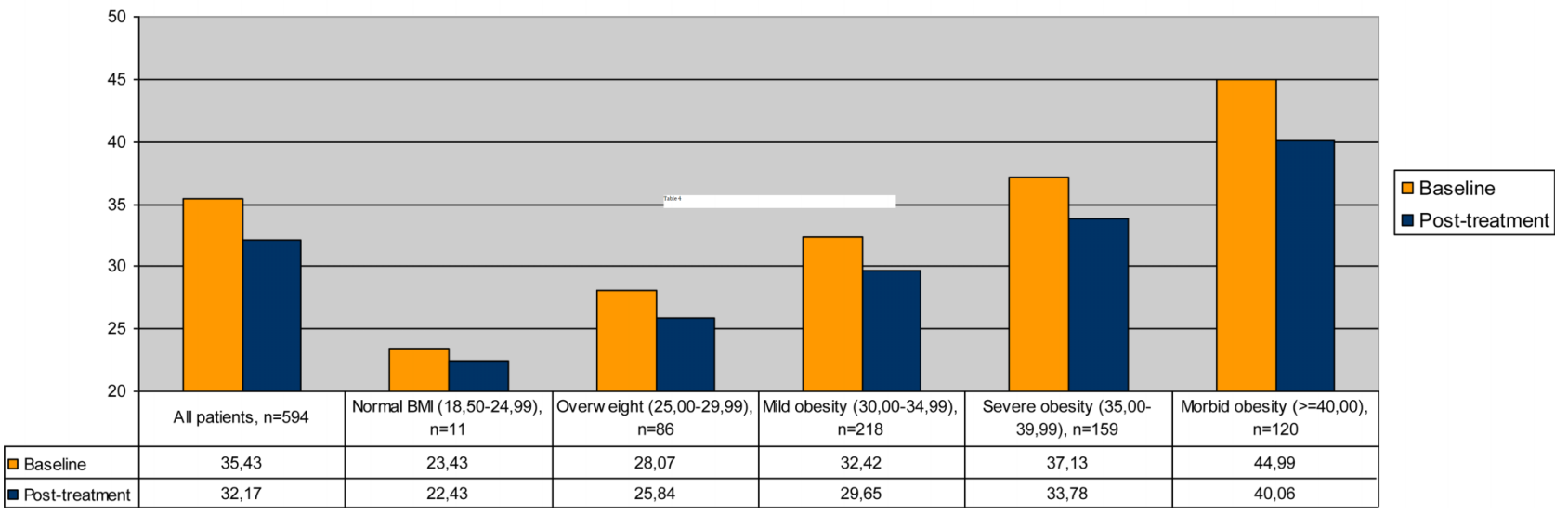


Table 2

Mean weight loss $(\mathrm{kg})$ after 1 year of self-empowerment group treatment (data for all patients and according to BMI category at baseline)

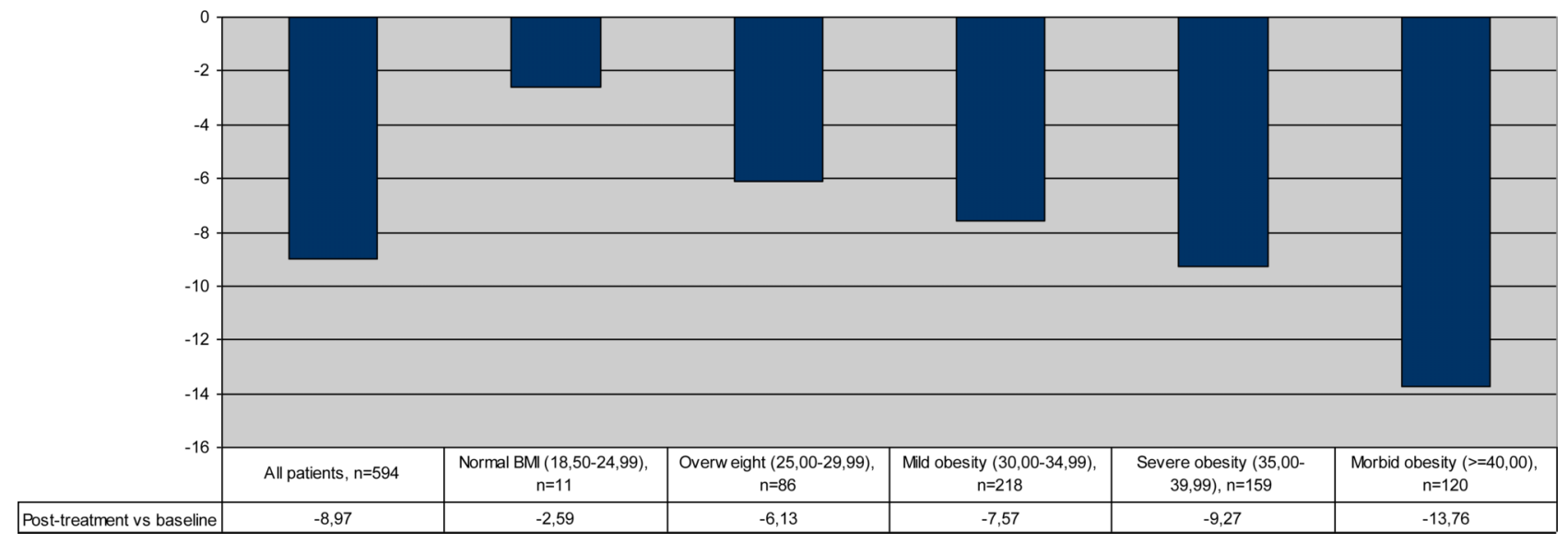

relationship of the person with his environment and with his facing the external world. ${ }^{20} 21$

The theoretical foundation of self-empowerment includes the following key attributes:

- The person as a value.

- The behaviour as a consequence of the personal perception of success or failure.

- The personal empowerment as a measure of change and of self-efficacy, allowing to pass from a perspective of need to a perspective of desire.

- The group as a space in which to activate these processes on the conscious level and 'here and now'.

- The group is also seen as a support, reference framework and a time/space allowing both identity and participation.

Recent research demonstrated that over the short term, overweight/obese persons can lose weight, whatever diet or programme they comply with. The problem is to maintain the weigh loss (and/or to continue losing weight) after the treatment programme is over. ${ }^{22-24}$
Overweight and obesity, as a result also of unhealthy lifestyles, can be addressed and tackled only if patients are determined to participate..$^{25}$ The persons reaching this service had already experimented with many diets, specialists' interventions, educational therapies, individual psychotherapies and even weight-loss surgery, with poor long-term effectiveness.

This therapy proposal was therefore worked out and tested as being different from the others, and mainly based on the motivational aspects of personality.

Our hypothesis is that self-empowerment helps people in improving quality of life both in terms of physical and psychical well-being and in terms of weight loss, maintaining the results achieved over time.

Before starting an effectiveness study and in order to standardise the procedures, a partnership was created with the Regional School for the Training in Primary Care that provided medical expertise to standardise health change evaluation. General practitioners in training were allocated within the mental health department

Table 3 Mean BMI in a subgroup of patients $(n=149)$ at baseline, after 1 year of selfempowerment group treatment and at 2006 follow-up evaluation

(data by year of group's start)

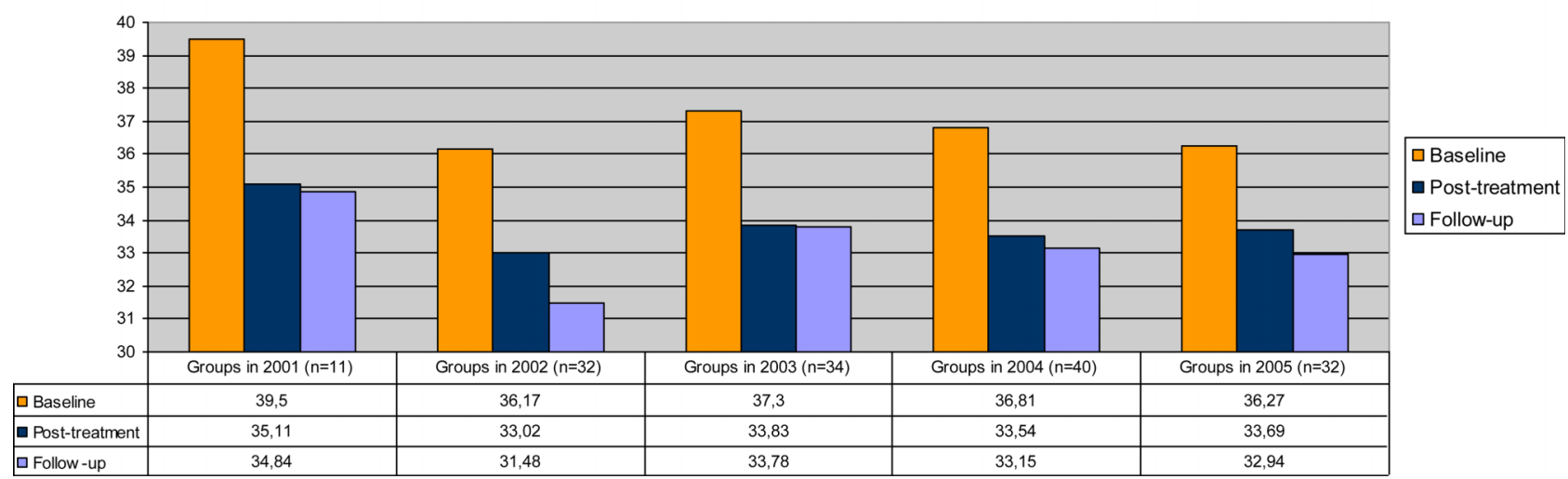


and given the responsibility to perform the clinical evaluation of the overweight and obese patients.

\section{Trial design}

This pilot study aims at observing, for a period of 1 year, the effects of individual empowerment on a set of qualitative (sense of coherence and well-being) and quantitative (clinical and laboratory) indicators in a group of overweight and obese persons. ${ }^{26}$

\section{Sample size}

A selected group of 80 overweight/obese patients will be included in the study. All highly motivated persons asking to enter the self-empowerment groups will be assessed for inclusion. The sample size is considered adequate to verify the research hypothesis.

\section{Inclusion criteria}

The study will include all adults (18 years and older), with a BMI $>25$, entering the new 'therapy groups' of the project. People will access the project either by

A. Spontaneous access through the 'Support Service' run by the Association or by

B. Referral to the service by general practitioners or specialists.

Before being included in the therapy groups, participants will undergo an inclusion phase of three individual in-depth interviews with an expert. The interviews are considered essential to assess patient's motivation to change (Likert scales $0-10$ on importance of the problem+self-confidence to change) and to strengthen their willingness to cope. Each of these aspects is in-depth evaluated in different sessions. A final interview, with request to sign the informed consent form and an agreement to follow the group sessions for 1 year, closes the inclusion phase.

\section{Exclusion criteria}

Minors, patients who must lose weight in a short time because of planned surgery, those who suffer from severe health problems (severe stroke outcomes, severe respiratory disease, heart or kidney failure, neoplasms and severe psychiatric pathologies), those who want to lose weight only for aesthetic reasons, those who cannot guarantee regular weekly attendance for the treatment and those who, at the same time, use other means for losing weight (weight-loss surgery, psychoanalysis or other nutritional regimens).

\section{Measures}

- Quantitative and qualitative measures will be collected.

- Main quantitative measures: body weight and waist circumference.

- Secondary quantitative measures: blood pressure, blood sugar levels, total blood cholesterol values, blood triglyceride values, creatine levels, benzodiazepines and antidepressants intake).
- In order to evaluate the presence of sleep apnoea and depression, frequently associated with obesity, the following questionnaires will be self-administered: Berlin sleep questionnaire ${ }^{27}$

- Hamilton rating scale for depression. ${ }^{28}$

- Qualitative measures: the following scales are used to translate complex qualitative issues, more linked to the salutogenic model of health into quantitative measures-Antonovsky's sense of coherence scale and a four-point Likert scale on empowerment. ${ }^{29-32}$

\section{Protocol of intervention}

Four groups of 20 people each will be created. The group meetings, lasting for $1.5 \mathrm{~h}$, will take place every week, always on the same day and at the same time, for 1 year. During each meeting, the participant 'weighs him/herself' and registers the weight in his/her daily diary. The same expert who performed the interviews will also be dedicated to the group conduction. Expert's major competences are psychotherapy, groups conducting and self-empowerment training.

The intervention is based on the following:

- Taking account of suffering living with over-running of 'personal internal killers'.

- Identification, activation, maximisation of personal strengths and resources.

- Adding and experimenting new possibilities through motivation to take action.

- Shifting from a needs-based life to a positive approach to life, based on pleasure and desire.

- Valorisation of successful experiences.

- Utilised methods are as follows:

- Written therapeutic agreement, to define cornerstones of the intervention.

- Circle time. ${ }^{33}$

- Brainstorming. ${ }^{34}$

- Daily diary. ${ }^{35}$

- The 'Daisy of Possibilities.'21

- Individual letters. ${ }^{35}$

\section{T 0 Baseline}

All patients will follow these procedures:

- Complete family, pharmacological history, cardiovascular risk assessment and physical examination, performed by a medical doctor.

- Computer-assisted filing (Excel spread sheet) of data: BMI, waist circumference, blood pressure (two measurements in 10-15 min) and non-invasive oximetry.

- Questionnaires administration: Antonovsky's sense of coherence, Berlin test on sleep apnoea and Hamilton Depression Test.

- Recording of existing blood test in the previous 3 months: blood cells count, glycaemia, thyroidstimulating hormone (TSH), total cholesterol, highdensity lipoprotein (HDL) cholesterol, urine test, glycate haemoglobin and creatine clearance.

- First group meeting with introducing the method.

- Handing over a personal daily diary. 
T2

- A 2-month follow-up.

- Meetings dealing with 'perceptive harmonisation' (getting to know one's own body) will be added to the group activities. This activity will consist of 10 weekly meetings led by experienced physiotherapists.

\section{T 6}

- A 6-month follow-up.

- The appointments will be agreed upon with the persons about 1 month in advance. A presentation letter for respective general practitioner will be prepared; participants will be invited to bring the results of any tests undergone under the previous 3 months.

- Physical examination plus Hamilton Depression Test questionnaire.

- Collection of narrative reflections about each personal life path.

- Participation in plenary meetings (all of the groups together) for 'knowledge sharing' with experts.

\section{$T 12$}

- Pharmacological intake evaluation, cardiovascular risk assessment and physical examination.

- Computer-assisted filing (Excel spread sheet) of data: BMI, waist circumference, blood pressure (two measurements in 10-15 min) and non-invasive oximetry.

- Questionnaires administration: Antonovsky's sense of coherence, Berlin test on sleep apnoea and Hamilton Depression Test.

- Recording of existing blood test in the previous 3 months: blood cells count, blood glucose, TSH, total cholesterol, HDL cholesterol, urine test, glycate haemoglobin and creatine clearance.

\section{Follow-up}

Participants will sign an agreement that they will participate in all the follow-ups. NGO's staff will call each patient to remind the importance of attending the sessions.

\section{Statistical analysis}

A descriptive analysis of all the parameters collected, both the baseline data and those from 6-month and 12-month follow-ups will be performed.

For continuous variables (age, weight, BMI, etc), indicators such as the mean, standard deviation, minimum, median and maximum values will be calculated. The categorical data will be presented by means of frequency tables $(\mathrm{n}, \%)$.

The changes in the parameters and in the distribution of categorical variables pertaining to those from the 6-month and 12-month follow-ups will be recorded. McNemar's test will be used on the categorical variables, with a level of significance for the $p$ value fixed at $<0.05$. This will allow the recording of any differences in the distributions of the main factors of interest. $\mathrm{T}$ tests will be used for paired data for assessing changes in the categorical variables (table 4 ).
Table 4 Synoptic table of the tools used

\begin{tabular}{llll}
\hline Tool & Month 0 & Month 6 & Month 12 \\
\hline $\begin{array}{l}\text { Daily diary } \\
\text { Form about motivation } \\
\text { for change }\end{array}$ & $\mathrm{X}$ & & $\mathrm{X}$ \\
$\begin{array}{l}\text { Form about } \\
\text { self-empowerment }\end{array}$ & $\mathrm{X}$ & & $\mathrm{X}$ \\
$\begin{array}{l}\text { Questionnaire about } \\
\text { emotional states }\end{array}$ & $\mathrm{X}$ & & $\mathrm{X}$ \\
$\begin{array}{l}\text { Questionnaire about } \\
\text { sense of coherence }\end{array}$ & $\mathrm{X}$ & & $\mathrm{X}$ \\
$\begin{array}{l}\text { Test about perceived } \\
\text { health }\end{array}$ & $\mathrm{X}$ & $\mathrm{X}$ & $\mathrm{X}$ \\
$\begin{array}{l}\text { Berlin test } \\
\begin{array}{l}\text { Audit of alcohol } \\
\text { consumption }\end{array}\end{array}$ & $\mathrm{X}$ & $\mathrm{X}$ & $\mathrm{X}$ \\
$\begin{array}{l}\text { Test for depression } \\
\text { Approval rating form }\end{array}$ & $\mathrm{X}$ & $\mathrm{X}$ & $\mathrm{X}$ \\
\hline
\end{tabular}

As drop-out rates are expected, particularly among nonrespondents, the potential for selection bias will be tested by comparing baseline patient characteristics between respondent and non-respondent. Indeed, an intention to treat approach will be used; this means that patients who will be lost to follow-up will be censored at the time of the last visit they attended and included into the analysis. Survival analysis techniques will be used by using Cox analysis for binary outcomes and generalised models for continuous variables. Missing data will be also handled by using the Last Observation Carried Forward method. ${ }^{36}$

\section{DISCUSSION}

Our assumption is that self-empowerment method can be a resource to enable people to manage stressful events, to discover external/internal resources, to mobilise them and to promote effective coping by finding solutions, in a health-promoting manner.

In overweight and obese patients, according to selfempowerment, losing weight is not the principal aim, but a desirable outcome of personal awareness and hopefulness.

We decided to evaluate not only physical and clinical parameters but also humanistic patient-oriented outcomes in order to stress the importance and novelty of this new methodology. ${ }^{37}$ In particular, the SOC questionnaire seems to be applicable for measuring people's ability to maintain health despite stress (coping), one objective of the self-empowerment approach.

At the end of this pilot study, we are expecting to identify a set of appropriate measures and procedures to determine the effectiveness of self-empowerment. Results will be recorded and analysed to start a randomised controlled trial to evaluate the effectiveness of the proposed methodology.

Acknowledgements All the authors acknowledge and are deeply grateful to the support and secretarial work given by Dr Mario Novello and Ms Giuliana Matellon ('Giulianete') of the Mental Health Department. 
Contributors PS and AC are the principal investigators who designed the study and drafted the article. AS and AT have made substantial contributions to the conception and design of the project; MB and FG have been involved in drafting the manuscript or revising it critically for important intellectual content; RF performed the dataset analysis, read and modified the text; LC and GT have read, reviewed and given the final approval of the version to be published. ST is in charge with the Statistical analysis.

Funding This research received no specific grant from any funding agency in the public, commercial or not-for-profit sectors.

Competing interests None.

Patient consent Obtained.

Ethics approval This protocol was approved by the local Ethics Committee of Udine in March 2012.

Provenance and peer review Not commissioned; externally peer reviewed.

\section{REFERENCES}

1. OECD. Health at a glance: Europe 2012. OECD Publishing. http:// www.oecd.org/els/health-systems/HealthAtAGlanceEurope2012.pdf (accessed 19 Mar 2013).

2. WHO. Obesity and overweight, Fact sheet No. 311, updated March 2013.

3. Portale dell'epidemiologia per la sanità pubblica a cura del Centro Nazionale di Epidemiologia, Sorveglianza e Promozione della Salute. http://www.epicentro.iss.it/problemi/obesita/obesita.asp (accessed 19 Mar 2013).

4. Istituto nazionale di statistica ISTAT 'Noi Italia 2012'. http://www.istat it/dati/catalogo/20120215 00/Noi_Italia 2012.pdf (accessed 19 Mar 2013).

5. Progetto cuore, coordinato da Istituto Superiore di Sanità. Epidemiologia e prevenzione delle malattie cerebro e cardiovascolari. http://www.cuore.iss.it/fattori/obesita.asp (accessed 19 Mar 2013).

6. Consultation, WHO. Obesity: preventing and managing the global epidemic. World Health Organization technical report series 894 2000.

7. Ekpe HI. Empowerment for adults with chronic mental health problems and obesity. Nurs Stand 2001;15:37-42.

8. http://www.epicentro.iss.it/problemi/obesita/obesita.asp (accessed 10 Mar 2013)

9. http://www.nhlbi.nih.gov/guidelines/obesity/ob home.htm (accessed 10 Mar 2013)

10. Gadde KM, David BA. Combination therapy for obesity and metabolic disease. Curr Opin Endocrinol Diabetes Obes 2009;16:353.

11. Leblanc ES, O'Connor E, Whitlock EP, et al. Effectiveness of primary care-relevant treatments for obesity in adults: a systematic evidence review for the U.S. preventive services task force. Ann Intern Med 2011;155:434-7.

12. Teixeira PJ, Silva MN, Mata J, et al. Motivation, self-determination, and long-term weight control. Int J Behav Nutr Phys Act 2012;9:22.

13. Rappaport J, Swift C, Hess R, eds. Studies in empowerment: steps toward understanding and action. New York: Haworth, 1984.
14. Swift C, Levine G. Empowerment: an emerging mental health technology. J Prim Prev 1987;8:71-94.

15. Gibson C. A concept analysis of empowerment. J Adv Nurs 1991;16:354-61.

16. Bruscaglioni M. Persona empowerment. Franco Angeli 2007:61-76.

17. Bruscaglioni M. Per una formazione vitalizzante. Franco Angeli 2005:175-224.

18. Aujoulat I, Luminet $O$, Deccache A. The perspective of patients on their experience of powerlessness. Qual Health Res 2007; 17:772-85.

19. Aujoulat I, Marcolongo R, Bonadiman L, et al. Reconsidering patient empowerment in chronic illness: a critique of models of bodily control and self-efficacy. Soc Sci Med 2008;66:1228-39.

20. Maddux JE, Rogers R. Protection motivation and self-efficacy: a revised theory of fear appeals and attitude change. J Exp Soc Psychol 1983;19:469-79.

21. Bruscaglioni M, Gheno S. II gusto del potere (the taste of power). Milano: Franco Angeli, 2000.

22. Wu T. Long-term effectiveness of diet-plus-exercise interventions vs. diet-only interventions for weight loss: a meta-analysis. Obes Rev 2009;10:313-23.

23. De Pue JD. Maintenance of weight loss: a needs assessment. Obes Res 2012;3:241-8.

24. Jeffery RW. Long-term maintenance of weight loss: current status. Health Psychol Off J Div Health Psychol Am Psychol Assoc 2000;19 (1 Suppl):5

25. Prochaska JO, Butterworth S, Redding CA. Initial efficacy of MI, TTM tailoring and HRl's with multiple behaviors for employee health promotion. Prev Med 2008;46:226-31.

26. Anderson JW, Elizabeth CK. Obesity and disease management: effects of weight loss on comorbid conditions. Obes Res 2012;9 (Suppl 4):326S-34S.

27. Ahmadi N. The Berlin questionnaire for sleep apnea in a sleep clinic population: relationship to polysomnographic measurement of respiratory disturbance. Sleep Breath 2008;12:39-45.

28. Luppino FS. Overweight, obesity, and depression: a systematic review and meta-analysis of longitudinal studies. Arch Gen Psychiatry 2010;67:220.

29. Lindstrom B, Eriksson M. Salutogenesis. J Epidemiol Commun Health 2005;59:440-2.

30. Antonovsky A. Health, stress and coping. San Francisco: Jossey-Bass, 1979

31. Antonovsky A. Unravelling the mystery of health. How people manage stress and stay well. San Francisco: Jossey-Bass, 1987.

32. Lindstrom B, Eriksson M. Professor Aaron Antonovsky (1923-1994) the father of the Salutogenesis. J Epidemiol Commun Health 2005;59:511.

33. Rogers C. Carl Rogers describes his way of facilitating encounter groups. Am J Nurs 1971;71.

34. Osborn AF. How to 'Think Up. New York, London: McGraw-Hill Book Co, 1942

35. Frattaroli J. Experimental disclosure and its moderators: a meta-analysis. Psychol Bull 2006;132:823-65.

36. Mallinckrodt $\mathrm{CH}$, Lane PW, Schnell D, et al. Recommendations for the primary analysis of continuous endpoints in longitudinal clinica trials. Drug Inf J 2008;42:303.

37. Ropka M. Symptom status and functional status outcomes: humanistic outcomes in obesity disease management. Obes Res 2002;10(Suppl 1):44s-8s. 